\title{
Linx
}

Revue des linguistes de l'université Paris X Nanterre

9 | 1997

Émile Benveniste. Vingt ans après

\section{La sémantique benvenistienne dans l'herméneutique de P. Ricœur}

\section{Grazyna Lubowicka}

\section{(2) OpenEdition}

\section{Journals}

Édition électronique

URL : http://journals.openedition.org/linx/1064

DOI : 10.4000/linx.1064

ISSN : 2118-9692

\section{Éditeur}

Presses universitaires de Paris Nanterre

\section{Édition imprimée}

Date de publication : 1 avril 1997

Pagination : 259-270

ISSN : 0246-8743

\section{Référence électronique}

Grazyna Lubowicka, «La sémantique benvenistienne dans l'herméneutique de P. Ricœur », Linx [En ligne], 9 | 1997, mis en ligne le 09 juillet 2012, consulté le 21 avril 2019. URL : http:// journals.openedition.org/linx/1064; DOI : 10.4000/linx.1064 


\title{
La sémantique benvenistienne dans l'herméneutique de P. Ricœur
}

\author{
Grazyna Lubowicka
}

$\mathrm{E}$ n quel sens pouvons-nous dire que la sémantique de Benveniste est présente dans la philosophie de P. Ricoeur?

Sans doute, est-elle un présupposé essentiel de la conception de la référence dans l'oeuvre littéraire que Ricoeur tâche d'élaborer dans la Métaphore vive ${ }^{1}$ ainsi que de la conception de la référence en termes de refiguration dans Temps et récit ${ }^{2}$ Mais, il y a aussi dans la philosophie ricœurienne, en particulier dans Soi-même comme un autre, une autre sorte de reprise des idées de Benveniste, à savoir, une discussion, une intégration et même une continuation de sa théorie des actes de discours et de la question de la subjectivité dans le langage.

L'objet principal de Soi-même comme un autre ${ }^{3}$ est l'herméneutique du soi, qui servira ensuite de base pour une ontologie et pour une éthique. Ainsi, le problème central sur lequel Ricoeur se concentre dans le livre est d'élaborer une conception du sujet, une conception telle qu'elle reste encore possible après toutes les références critiques de la philosophie contemporaine.

$\mathrm{Si}$, dès lors, nous voulons prendre pour tâche de déterminer la position de la sémantique benvenistienne dans la philosophie de P. Ricœur, notre enquête va se concentrer sur l'herméneutique du soi telle quelle est développée dans Soi-même comme un autre, dans laquelle la question du sujet reste une question centrale et primordiale. Cette perspective de l'herméneutique ricœurienne nous permettra, comme nous l'espérons, de déceler et de caractériser le lieu que la pensée de Benveniste occupe en dehors du domaine de la linguistique, dans la philosophie contemporaine, et, en particulier, dans sa problématique la plus troublante qui est celle de la subjectivité.

\footnotetext{
1 P. Ricoeur, La Métaphore vive, Paris, Ed. du Seuil, 1975.

2 P. Ricoeur, Temps et Récit, tome I-III, Paris, Ed. du Seuil, 1975, 1989, 1984.

3 P. Ricoeur, Soi-même comme un autre, Paris, Ed. du Seuil, 1990.
} 


\section{Grazyna Lubowicka}

En effet, le problème qui nous paraît le plus intéressant et le plus visible dans cette perspective, le problème ressortissant à la fois à la linguistique de Benveniste et à l'herméneutique de P. Ricœur, est celui de l'auto-identification ou de l'auto-désignation, du sujet parlant par ses actes de discours. L'identification ou bien l'individualisation sont des termes philosophiques déterminant les procédures langagières spécifiques de désignation, distinctes de la prédication et visant l'individu. Ainsi, c'est le problème de la désignation de l'individu qui sera notre fil conducteur le long de cette enquête. Il est essentiel pour l'herméneutique, puisqu'il en constitue le premier pas, la voie d'accès à la problématique du soi ; mais, pour la sémantique benvenistienne il est également important dans la mesure où il permet d'ouvrir toute une série d'interrogations touchant le sens même et le statut des termes sujet de l'énonciation et suiréflexivité. C'est donc, en général, le problème de la subjectivité dans le langage qui constitue ce noyau commun, ce lieu d'échanges, entre la linguistique et la philosophie. Dans les deux cas, en effet, c'est le langage et non pas les catégories d'être ou de substance qui est le fondement et le point de départ.

\section{Ricœur et Benveniste devant l'actuel}

L'herméneutique, suivant Soi-même comme un autre, est une approche indirecte du soi à travers l'interprétation de son agir; elle se révèle donc comme une philosophie du détour, où la réflexion est entièrement médiatisée par l'analyse. La voie longue de l'articulation de la réflexion sur l'analyse, choisie par Ricœur, non seulement est une nécessité imposée par la position indirecte du soi, mais elle a aussi son aspect positif, à savoir, elle permet à l'herméneutique de restituer une dimension critique et de s'approcher d'une description objective ou objectivante du soi.

Afin de marquer sa distance à la fois des philosophies du cogito et de celles de sa destitution, Ricoeur propose, dans son herméneutique, de remplacer le je de la première personne par le soi, index réflexif qu'il appelle omni-personnel. Le soi dans son usage philosophique est réflexif de toutes les personnes grammaticales (je, tu, il), et même des pronoms impersonnels (chacun, quiconque, on). Privé de sa prétention autofondatrice, (propre au je des philosophies du cogito), inaccessible à l'évidence de l'intuition immédiate, le soi se voit doté d'une fragilité spécifique : le je se pose ou est déposé, le soi est impliqué à titre réfléchi dans les opérations dont l'analyse précède le retour vers lui-même; le je revendique une unité et une simplicité indécomposable, le soi ne peut prétendre qu'à l'identité au sens d'ipséité. La fragilité du soi s'exprime également dans le caractère forcément problématique de toutes les études herméneutiques. Le soi, enfin, est inséparable et structuré immédiatement par l'altérité.

Refuser l'ambition de fondation ultime du Cogito, ce n'est pas abandonner la problématique de la subjectivité ; bien au contraire, la structure du sujet humain, son éthique, son ontologie, restent au centre du travail philosophique de Ricoeur. Aussi l'intention de Ricoeur sera-t-elle de restituer la problématique 
du sujet, bien que toute la philosophie actuelle soit frappée par la crise $d u$ cogito, et malgré l'ensemble de la critique entreprise par celle-ci. Ni cogito exalté, ni cogito humilié, dit Ricoeur ; l'herméneutique du soi voudrait être une troisième voie à distance ou au-delà de l'apologie du cogito et de sa destruction.

Le seuil du XXème siècle est également l'époque de la naissance de la linguistique. En conséquence, à partir de ce moment, la philosophie est placée devant la question du langage, promue constamment tant par la philosophie analytique que par la linguistique française. Ce qui est pourtant spécifique à cette dernière, c'est que cette promotion de la question du langage a pris la forme de la domination du modèle sémiologique, érigé ensuite, (résultat des courants structuralistes), en paradigme général de rationalité. Le modèle ainsi élaboré était donc une conception du langage qui excluait expressément, quoique pour des raisons purement méthodologiques, la question de la référence et de la subjectivité du domaine de sa recherche. Ce problème n'a pris une acuité particulière que sur le sol de la philosophie française, car ni la tradition anglo-saxonne, ni la tradition allemande, n'ont éliminé la question de la référence et du sujet de façon aussi radicale.

Le tournant linguistique et la querelle $d u$ cogito, les deux questions les plus inquiétantes de la pensée actuelle, constituent également un défi pour Ricoeur, pour lui-même et pour sa propre conception philosophique. Cependant, essayer de reprendre la problématique du sujet après avoir intégré l'ensemble de la critique de la philosophie contemporaine, c'est, avant tout, abandonner le fondement stable du cogito moderne avec son ambition à se connaître d'une façon immédiate. C'est pourquoi le je doit être remplacé par le soi. C'est pourquoi l'approche herméneutique est forcément fragmentaire et rhapsodique ; c'est pourquoi, enfin, la réflexion doit être médiatisée par l'analyse.

Concernant les sciences du langage, la position de Ricœur semble également particulière. Loin d'accepter sans réserve le modèle sémiologique, mais aussi loin de chercher à éviter une rencontre avec les sciences du langage, Ricoeur propose d'engager une conversation triangulaire entre la linguistique, la philosophie analytique et la philosophie du langage, une conversation qui, plus tard, dans Soi- même comme un autre, se précisera comme la tâche d'intégrer la philosophie du langage dans la philosophie herméneutique. L'intention de Ricœur sera, dès lors, de conjoindre les deux traditions hétérogènes et à première vue inconciliables : les sciences du langage d'une part et l'herméneutique du soi de l'autre. Ainsi, en dernière instance, la philosophie du langage joue dans l'herméneutique ricœurienne le rôle de propédeutique à la question de l'ipséité, la sémantique frégéenne et la pragmatique, constituant le premier pas, la voie d'accès à l'herméneutique du soi. En effet, le soi conçu tant comme le je -tu que comme la troisième personne doit se prêter à une description double, à savoir, il doit être accessible aussi bien comme je, sujet parlant et réfléchissant que comme $i l$, objet de la description objective, chose parmi des choses. C'est donc toujours le seul et même soi qui est tantôt capable de se désigner lui-même comme auteur de ses propres actions, tantôt de se laisser désigner de manière objective. 


\section{Grazyna Lubowicka}

La première étape de l'herméneutique se divise donc en deux voies parallèles introduisant à la problématique du soi, à savoir, la voie de la référence identifiante où le soi est désigné de façon objective et 1a voie de la théorie de l'énonciation ou le soi est capable de se désigner lui-même en parlant. Les deux voies ensemble contribuent à construire "une théorie du soi au niveau linguistique", servant ensuite de base à l'ontologie et à l'éthique. Mais, il est bien visible que les deux voies se fondent sur le modèle du langage intégrant la dimension du sens et de la référence.

Aux yeux de Ricoeur, c'est bien Benveniste qui, avec sa linguistique de la phrase, a réintroduit la question de la référence, après la coupure opérée par la sémiologie de Saussure. La présence de la sémantique benvenistienne dans l'herméneutique ne se réduit pourtant pas au postulat de la référence. Il y a sans doute quelque chose de plus. La sémantique benvenistienne, plus précisément, la théorie réflexive de l'énonciation, fait partie en effet, à côté d'autres sciences du langage, de ce premier pas de la théorie ricœurienne du soi sur le plan linguistique.

\section{L'approche de la personne par la voie de la référence identifiante}

Les deux voies d'accès à la problématique du soi, la voie par la référence identifiante et la voie par la théorie réflexive de l'énonciation révèlent leur importance pour l'herméneutique en ceci qu'elles peuvent lui offrir des procédures linguistiques permettant d'identifier une personne. Afin de voir mieux le rôle de la théorie de l'énonciation et pour marquer quel enrichissement de la notion de personne grâce à elle devient possible, nous commençons par la voie de la référence identifiante, relevant de la sémantique frégéenne.

L'identification (ou l'individualisation), repose sur des procédures langagières spécifiques de désignation, visant l'individu, non répétable et distinct de tous les autres de la même classe. Identifier, à ce stade, sur la voie de la référence identifiante, c'est donc pouvoir faire connaître à autrui, au sein d'une gamme des choses particulières du même type, celle dont nous avons l'intention de parler. En fait, le langage se composant pour une grande partie de concepts n'est pas pour autant démuni d'instruments permettant d'appréhender l'individu. Il est possible, en particulier, de distinguer trois procédures visant à désigner l'individu à l'exclusion de tous les autres: les noms propres, les descriptions définies et les indicateurs tels que pronoms personnels $(j e, t u, i l)$, démonstratifs (ceci), adverbes de lieu, de temps, de manière, temps verbaux.

L'individu humain dans l'approche sémantique n'est pourtant aucunement privilégié, de même que ne le sont pas les indicateurs je, $t u$. Identifier à ce stade, ce n'est en effet qu'identifier quelque chose en général, pas identifier exclusivement une personne, pas plus que s'identifier soi-même. 
La personne visée par la voie de la référence identifiante est donc entendue comme une chose en général, si ce dont nous parlons est une chose et pas comme un sujet capable de se désigner lui-même en parlant. L'identification référentielle n'attache non plus aucun privilège aux indicateurs $j e, t u$, dans la mesure où ils gardent pour point de repère, de même que les autres déictiques, l'énonciation entendue ici comme événement arrivant dans le monde objectif, c'est-à-dire sans lien avec un sujet parlant.

Quelle notion de personne se laisse déduire à l'issue de la voie de la référence identifiante? En d'autres termes, quel est le statut de la personne désignée par les opérateurs d'identification?

La personne ainsi déduite ne se comprend qu'en un sens très pauvre du mot, c'est-à-dire comme une chose dont nous parlons. La personne se situe donc à côté des choses, à côté des entités composant le monde objectif. C'est un objet de discours et non un sujet parlant, c'est une troisième personne, le lui de Benveniste, sans capacité de s'identifier soi-même.

Et pourtant, l'approche référentielle n'est pas sans importance pour l'herméneutique du soi, bien au contraire ; à partir de la notion de particulier de base, issue de la théorie de P. Strawson ${ }^{4}$, la personne peut en effet se définir comme référent unique auquel il est possible d'attribuer deux sortes de prédicats : des prédicats psychiques et physiques. En conséquence, la personne se comprend aussi comme un corps parmi des corps objectifs composant le monde, et elle s'inscrit sur le plan public, étant ainsi susceptible d'une description objective. Cette idée du corps propre appartenant à la fois au monde objectif des corps et à un individu comme le sien, comme un aspect du soi, restera présente jusqu'à la fin de l'enquête de Ricoeur.

Ce qui, dans l'approche strawsonienne, est pourtant de la plus grande importance, c'est que les prédicats physiques et psychiques gardent la même signification qu'ils soient attribués à un soi ou à un autre que soi, à une troisième personne, à quiconque, à chacun. Strawson dit qu'ils sont selfascrivable ou other-ascrivable. En conséquence, les prédicats, même purement psychiques, se prêtent à une attribution objective, sur le plan public, à quelqu'un, à chacun, à une troisième personne. "Il faudra, dit Ricoeur, garder la force de ce $\operatorname{chacun}^{5}$ », qui n'est rien d'autre que le lui auquel Benveniste refusait le statut de personne.

Cependant, l'approche référentielle, en accentuant la troisième personne, occulte par cela même la question du soi qui sera donc à chercher plutôt du côté de l'autodésignation du sujet parlant et ne sera mis en lumière qu'au terme de la théorie réflexive de l'énonciation. Il ne s'agit pourtant pas de

\footnotetext{
4 P. Strawson, Individuals, Londres Methuen and Co, 1959 ; trad. fr. d'A Shalom et P. Drong, Les Individus, Paris, Ed. du Seuil, 1973.

5 P. Ricoeur, Soi-même comme un autre, p. 49.
} 


\title{
Grazyna Lubowicka
}

méconnaître ou de mépriser les avantages de la voie de la référence identifiante. L'enjeu de Ricoeur sera précisément de conjuguer la force logique de la troisième personne avec le pouvoir d'auto:désignation qui fait de la personne un soi.

\begin{abstract}
«Ce sera plutôt un problème pour nous de comprendre comment le soi peut être à la fois une personne dont on parle et un sujet qui se désigne à la première personne tout en s'adressant à une seconde personne. Ce sera un problème, car il ne faudra pas qu'une théorie de la réflexivité nous fasse perdre le bénéfice certain de la possibilité de viser la personne comme une troisième personne, et non pas seulement comme un je et un tu. La difficulté sera plutôt de comprendre comment une troisième personne peut être désignée dans le discours comme quelqu'un qui se désigne soi-même comme première personne. Or, cette possibilité de reporter l'autodésignation en première personne sur la troisième, aussi insolite soit-elle, est sans doute essentielle». ${ }^{6}$
\end{abstract}

\section{L'approche de la personne par la voie de la théorie réflexive de l'énonciation}

Rappelons qu'au terme de la première voie ressortissant à la philosophie du langage la personne apparaissait comme un particulier de base, irréductible à tout autre, comme un lui dont on parle et auquel on attribue des prédicats psychiques et physiques. Le soi, considéré comme la troisième personne grammaticale était encore, à ce stade, privé de sa propriété la plus essentielle, celle de l'ipséité, entendu au sens fort de la conscience de soi, quoique, comme le remarque Ricoeur, elle n'y soit nullement absente. Or, tant l'idée de corps propre supposant en quelque sorte une possession, que l'idée d'attribution en termes d'ascription, impliquent un sujet capable de se dire posséder son corps, de s'ascrire en un sens spécifique des prédicats psychiques, bref, de se désigner soi-même.

Quel enrichissement de la notion de personne peut-on recevoir de la théorie réflexive de l'énonciation? Autrement dit, quelle est la contribution de la théorie de l'énonciation à une théorie du soi ? Quel est le rôle de Benveniste, lui-même, dans cette interrogation ?

La théorie de l'énonciation qui inclut l'approche benvenistienne avec son prolongement chez J. L. Austin ${ }^{7}$ et chez J. R. Searle ${ }^{8}$, est située par Ricoeur dans le cadre de la pragmatique logique au sens de Morris où elle se définit comme étude de l'emploi effectif du langage dans tous les cas où la signification d'une

\footnotetext{
${ }^{6}$ Ibid, p. 48.

7 J. L. Austin, How to do things with words, Harvard University Press, 1962, trad. fr. de G. Lane, Quand dire, c'est faire, Paris, Ed. du Seuil, 1970.

8 J. R. Searle, Les Actes de la langage, Paris, Hermann, 1972.
} 
proposition varie en fonction du contexte d'interlocution. La pragmatique met au centre de la problématique non plus l'énoncé mais l'énonciation ou l'acte même de dire qui désigne réflexivement son locuteur, impliquant simultanément un je qui parle et un $t u$ auquel le premier s'adresse, la troisième personne étant exclue comme non-personne, selon l'expression de Benveniste.

L'approche par la théorie de l'énonciation met ainsi directement en scène les trois éléments constitutifs à la théorie du soi : le je, le $t u$, la situation de discours et le modèle du langage intégrant le sens et la référence. Quelle nouveauté apporte-t-elle par rapport à l'approche sémantique ?

Tout d'abord, c'est le pronom je qui est promu au premier rang des indicateurs. Le je qui, auparavant, pris en dehors du rapport de réflexivité de l'énonciation ne présentait aucun ordre privilégié, maintenant, mis en rapport avec l'acte de dire, devient le premier des indicateurs, le pivot autour duquel les autres se regroupent. Le je indique toute personne qui en parlant se désigne elle-même, entraînant à sa suite le $t u$ de l'interlocuteur. «Je, comme le dit Benveniste, se réfère à l'acte de discours individuel où il est prononcé et il en désigne le locuteur ${ }^{9}$ ». «La subjectivité est la capacité du locuteur de se poser comme sujet» 10

L'approche benvenistienne introduit donc une personne qui a la capacité de se désigner soi-même en parlant. C'est, dès lors, aux yeux de Ricoeur, l'idée d'identification en termes d'autodésignation qui constitue la découverte principale de Benveniste. Le sujet de la théorie des actes de discours se réduit donc à un seul trait, à un seul geste, à ce mouvement vers lui-même, à l'acte d'autodésignation. La subjectivité impliquée dans la théorie de Benveniste n'est rien d'autre que la capacité d'autoréférence. Cette propriété, encore absente dans l'approche référentielle, n'est attribuable, selon Ricoeur, qu'à soi compris comme ipséité.

Deux problèmes sont donc visibles dans la théorie benvenistienne de l'énonciation: celui de la subjectivité dans le langage et celui de la réflexivité de l'énonciation. Mais les deux problèmes, posés ensemble et étudiés dans le contexte de l'herméneutique du soi, ouvrent toute une série de doutes et même d'apories concernant particulièrement le lien entre l'acte d'énonciation et le sujet de l'énonciation.

A première vue, le rapport entre l'énonciation et son auteur ne paraît pas faire problème. L'implication de l'énonciateur dans l'énonciation, dit Ricoeur, n'est-elle pas décelée sans ambiguiité par la possibilité d'adjoindre la formule développée des performatifs explicites - j'affirme que, j'ordonne que, je promets $q u e$ - à tous les actes illocutoires ? N'est-ce pas dans ce préfixe même que le je est marqué et n'est-ce pas à travers ce préfixe que le je atteste sa présence en toute énonciation?

\footnotetext{
${ }^{9}$ E. Benveniste, Problèmes de linguistique générale, tome II, Paris, Gallimard, 1974, p. 254.

10 Ibid, p. 259.
} 


\section{Grazyna Lubowicka}

Cependant, l'examen ricoeurien de ces problèmes, opéré dans la perspective de sa propre tâche, montre que non seulement le je est frappé d'une ambiguïté, mais aussi que le statut du sujet de l'énonciation et le sens même de sui-réflexivité, sont chargés de paradoxe. Les paradoxes décelés par l'enquête ricoeurienne constituent une sorte de résumé des interprétations divergentes de la théorie des actes de discours, en les rassemblant, en dernière instance, sous la forme d'une aporie.

Tout d'abord, il est bien visible que ce ne sont pas les énoncés, ni même les énonciations qui réfèrent, mais les sujets qui parlent et qui, en parlant, usent des ressources du sens et de la référence de l'énoncé pour échanger leur expérience dans une situation d'interlocution. Le locuteur en parlant s'approprie la langue entière, selon l'expression de Benveniste, et il la remplit du sens et de la référence à partir de lui-même, de son point de vue, s'annonçant par cela même comme pivot de référence, comme centre de perspective sur le monde.

Et pourtant, la théorie de l'énonciation est aussi bien concevable sans la nécessité de faire référence à un sujet. La réflexion se manifeste dans ce cas simplement comme un facteur d'opacité, lequel, selon la formule de F. Récanati11, interfère avec la transparence présumée d'un sens qui, sans lui, se laisserait traverser par la visée référentielle. Ce paradoxe de la réflexivité en tant qu'opacité, reconnu d'ailleurs déjà par les Anciens, est propre à toutes les énonciations dans la mesure où elles sont traitées comme actes, et il consiste précisément en ceci que la réflexion du fait de l'énonciation fait partie intégrante de sa référence. Toute énonciation par le fait même de l'énoncer, contient donc en soi un surcroît de sens, interférant avec sa référence, et par conséquent, toute énonciation peut être dite réflexive ou performative. Chaque énoncé en tant qu'acte de discours, dit Récanati, se pose comme ayant telle ou telle force illocutoire et fait donc réflexion sur lui-même de façon explicite ou implicite.

C'est dans ce type d'énoncés que le je est porté à l'expression, les performatifs n'ayant la vertu de faire-en-disant qu'exprimés par les verbes à la première personne, souvent non dits, mais qu'on peut expliciter en faisant précéder l'énoncé par le je de la forme j'affirme que. Le préfixe incluant je des performatifs explicites devient ainsi modèle pour l'expression linguistique de l'élément illocutoire de tous les énoncés.

Mais, cette réflexivité opacifiant le sens d'un énoncé se rapporte-t-elle encore à un sujet ? Or, c'est précisément l'acte et non l'agent et, dans l'acte, son élément illocutoire qui constitue le terme maître de la théorie des actes de discours. La réflexivité est ici attribuable, remarque Ricoeur, non à un sujet mais au fait même de l'énonciation, suivant la formule de Récanati : «dans le sens d'un énoncé se réfléchit le fait de son énonciation» ${ }^{12}$.

\footnotetext{
11 F. Récanati, La Transparence et l'Enonciation, Paris, Ed. du Seuil, 1979.

12 Ibid, p. 7.
} 
Une telle déclaration identifie donc la réflexivité à la sui-référence de l'énonciation, et, en conséquence, l'énonciation à laquelle celle-ci est attachée, est traitée comme un fait, autrement dit comme un événement qui se produit dans le monde. L'acte d'énonciation est devenu un fait qui survient dans le même monde que les faits et les états de choses visés référentiellement par l'énoncé. Bref, la réflexivité se ramène à la factualité même qui opacifie l'énonciation.

Nous avons à faire ici, constate Ricoeur, à une réflexivité qui n'est pas intrinsèquement liée à un soi, autrement dit, à un paradoxe d'une réflexivité sans ipséité, à un se sans soi-même. A travers la formule «dans le sens d'un énoncé se réfléchit le fait de son énonciation", dit-il, l'expression "se réfléchit» pourrait tout aussi bien être remplacée par «se reflète». Ainsi, on confère à l'énoncé deux références de direction opposée, une référence vers la chose signifiée et une référence vers la chose signifiante, autrement dit une suiréférence et une référence ad extra.

Mais, la question se pose, la réflexivité et la sui-référence de l'énonciation sont-elles deux notions équivalentes ? Le je, se demande Ricoeur, qui réfère et signifie ne disparaît-il pas ? Comment un acte d'énonciation opéré par un sujet peut-il se réduire à une chose, à un événement, à la factualité ? Plus gravement, comment le sujet qui réfère peut-il se désigner comme chose tout en restant un sujet ? La réflexivité n'est-elle pas quelque chose de plus que l'autoréférence de l'énonciation?

Comme nous le voyons, la divergence des interprétations de la réflexivité du langage se transforme, chez Ricoeur, en aporie, plus précisément, en aporie du sujet parlant qui n'est visé qu'obliquement comme l'expression marquée à l'intérieur d'une énonciation et liée avec l'acte d'énonciation, traité comme un fait arrivant dans le monde, dans le même monde que les choses auxquelles nous faisons référence.

Autrement dit c'est une aporie du sujet qui est traité d'une part comme un fait appartenant au monde et d'autre part comme centre de référence, comme point limite et non comme un de ses contenus. Enfin, c'est aussi le pronom je qui est frappé d'une ambiguïté, le je se manifestant à la fois comme un terme vacant, comme un shifter qui, à ce titre, désigne quiconque le prononce hic et nunc, et comme un je ancré, rivé à une unique perspective sur le monde et, pour cette raison, insubstituable, ne désignant que le seul moi. Aussi, selon Ricoeur, tous les paradoxes inhérents à la théorie réflexive de l'énonciation se laissent-ils résumer sous la forme d'une aporie ultime, de l'aporie étudiée depuis longtemps par L. Wittgenstein ${ }^{13}$, dite aporie de l'ancrage. L'aporie concerne autant le sujet parlant que le pronom je, et elle consiste en

\footnotetext{
13 L. Wittgenstein, Tractatus logico-philosophicus, trad. fr. de P. Klossowski, Paris, Gallimard, 1961.

- Le Cahier bleu et le Cahier brun, trad. fr. de P. Klossowski, Paris, Gallimard, 1961.

- Investigations philosophiques, trad. fr. de P. Klossowski, Paris, Gallimard, 1961.
} 


\section{Grazyna Lubowicka}

une contradiction, en un fossé logique entre le je, limite du monde et le je désignant une personne réelle, existant effectivement dans le monde, comme l'atteste l'assignation d'un nom propre au porteur du discours :

"L'aporie restait occultée, dit Ricoeur, dans l'interprétation selon laquelle le renvoi réflexif se faisait moins à l'ego de l'énonciation, traité comme un événement de monde, qu'au fait même de l'énonciation [...] Cette interprétation n'est plus possible, dès lors que l'on prend en considération l'aporie de l'ancrage»14.

\section{L'entrecroisement de deux voies de la philosophie du langage}

L'aporie de l'ancrage, suscitée à la fin de l'examen du statut du sujet dans le langage et de la notion de sui-réflexivité, contient pourtant un enjeu philosophique. Résoudre cette aporie, ce sera également pour Ricoeur achever un dernier pas dans la tâche de construire une théorie intégrée du soi sur le plan linguistique, dans la tentative de saisir et comprendre le soi à la fois comme ipséité référant et comme personne objective, référée. Une sortie possible de l'aporie consisterait, d'après Ricoeur, dans l'entrecroisement de deux voies de la philosophie du langage.

$\mathrm{Si}$, en effet, la première voie conduit vers le soi comme particulier de base, vers le lui appartenant au monde objectif et si la seconde fait apparaître le je-tu de la situation de l'interlocution à l'exclusion de la troisième personne, alors il faudra maintenant chercher à opérer en sens inverse une assimilation entre le je qui parle et le lui, posé parmi des choses dont nous parlons. La question sera finalement, dit Ricoeur, comment le je-tu de l'interlocution peut s'extérioriser dans un lui sans perdre la capacité de se désigner soi-même et comment le il-elle de la référence identifiante peut s'intérioriser dans un sujet qui se dit lui-même :

«La notion de sui-référence dont on suspectait plus haut la cohérence, est en fait le mixte issu du recroisement entre réflexivité et référence identifiante». ${ }^{15}$

La procédure linguistique, à partir de laquelle cette conjonction de la réflexivité et de la référentialité peut être opérée est fournie par le modèle d'inscription du temps phénoménologique sur le temps cosmologique, le modèle analysé déjà par Ricoeur, dans Temps et Récit étant très proche de l'idée benvenistienne de temps chronique.

Ainsi, cette corrélation entre le présent vif et un instant quelconque, par la construction du calendrier qui détermine la suite des dates possibles, a son

\footnotetext{
14 P. Ricoeur, Soi-même comme un autre, p. 68.

15 Ibid, p. 69.
} 
parallèle dans la désignation du ici absolu, déterminé par notre propre corps avec un des lieux possibles, repérés objectivement et, également, dans la conjonction entre le sujet, limite du monde et une personne, objet de référence identifiante. L'inscription dans ce cas repose sur la force illocutoire d'un acte de discours particulier, sur l'acte d'appellation. C'est le nom propre qui assume cette fonction de fixer l'identité d'un individu et de l'ancrer dans le monde. Le je ainsi inscrit et le lui, porteur du nom propre veulent dire la même personne. «Ce n'est donc pas arbitrairement, dit Ricoeur, que la personne, objet de référence identifiante et le sujet, auteur de l'énonciation, ont même signification ; une inscription d'un genre spécial, opérée par un acte spécial d'énonciation, l'appellation, opère la conjonction» ${ }^{16}$.

Au terme de l'enquête ricoeurienne, à l'issue de l'entrecroisement des deux voies de la philosophie du langage, nous recevons donc le soi se prêtant à une identification double, le soi apparaissant à la fois comme personne objective, référée et comme sujet réfléchissant, se désignant lui-même. En bref, le soi capable de se désigner lui-même est dit le même que l'une des personnes existant effectivement dans le monde.

La théorie du soi au niveau linguistique n'est toutefois que le premier pas, la voie d'entrée à la question du soi; l'herméneutique ricoeurienne embrasse également la sémantique de l'action (niveau praxique), et elle s'enrichit ensuite d'une éthique et d'une ontologie.

En sortant de la philosophie du langage, nous pouvons encore poser des questions sur les perspectives qui s'ouvrent grâce à cette conjonction de la linguistique et de l'herméneutique ; sur la sorte d'être qui permet cette double procédure d'identification comme personne-objet et comme sujet se désignant soi-même.

C'est, tout d'abord, à une réflexion de caractère ontologique sur le phénomène du corps propre que la philosophie du langage nous invite. Cette réflexion s'annonçait déjà à l'occasion de la théorie strawsonienne des particuliers de base où le corps propre apparaissait comme ayant à la fois le statut de la réalité physique et observable et celui de l'appartenance à un soi ; maintenant, au terme de l'enquête pragmatique, elle s'identifie à l'idée du corps propre manifestant la même structure mixte que je-tu, $c^{\prime}$ est-à-dire en tant que corps parmi le corps constituant un fragment de l'expérience du monde, et en tant que mien partageant le statut du je, limite du monde. A son tour, la corporéité fait partie d'une structure plus vaste, à savoir le problème de l'appartenance sur le mode corporel à ce que Husserl dénomme Lebenswelt et Heidegger In-der-Welt-Sein. (laissons le dernier mot à Ricoeur lui-même).

"L'ontologie de la corporéité et de l'être au monde resterait une prétention inconsistante si, dit-il, elle n'était pas conceptuellement articulée dans une

\footnotetext{
16 Ibid, p. 71.
} 


\section{Grazyna Lubowicka}

sémantique et une pragmatique, de telle façon que la double allégeance du corps propre au monde des événements et à celui du soi puisse se réfléchir, se refléter, dans les procédures de langage qui relient la personne comme quelque chose dont on parle et le soi impliqué réflexivement dans les actes de discours.»17

17 P. Ricoeur, Attestation, dans : Paul Ricoeur - Les métamorphoses de la raison herméneutique, publié sous la direction de . J. Greisch et R. Kearney, Paris, Cerf, 1991, p. 389. 